\title{
Impact of Clinical Pharmacist Interventions on Potentially Inappropriate Medications in Elderly Primary Care Patients With Mental Disorders Using Polypharmacy: A Cohort Retrospective Study
}

Matej Štuhec ( $\square$ matejstuhec@gmail.com )

University of Maribor

Alja Nemec

University of Ljubljana

\section{Research Article}

Keywords: elderly patients, mental disorders, potentially inappropriate medication, clinical pharmacist, medical review.

Posted Date: January 4th, 2021

DOl: https://doi.org/10.21203/rs.3.rs-131367/v1

License: (c) (1) This work is licensed under a Creative Commons Attribution 4.0 International License.

Read Full License 


\section{Abstract}

Potentially inappropriate medications (PIMs) are highly prevalent in elderly patients with mental disorders (MPHs) treated with polypharmacy. This study's main aim was to determine the impact of a clinical pharmacist (CP)'s recommendations on the total number of PIMs and to evaluate the differences in PIMs between two patient subgroups with mental disorders with either fully or partially accepted recommendations that have not been studied until now.

A retrospective observational cohort study involving primary care patients aged 65 or above in 20152017 was conducted. To evaluate the impact of the interventions on the number of PIMs, odds ratios (ORs) were used. Ninety-nine patients were included (79.4 y, SD=7.92). At least one PIM was present in $69.7 \%$ or $92.9 \%$ of the patients (PRISCUS and Beers list). The interventions led to a $21.2 \%$ decrease ( $n=23$; PRISCUS) and a $17.3 \%$ decrease $(n=38 ;$ Beers $)(p<0.05)$. The ORs for PIMs were significantly smaller in the full-acceptance subgroup ( $\mathrm{OR}=3.8, \mathrm{Cl} 1.4-10.1$; Beers) and insignificantly smaller for another group (OR=2.8, $\mathrm{Cl} 1.0-8.2$; PRISCUS). The CP's interventions decreased the number of PIMs according to the PRISCUS and the Beers list, and ORs for PIMs decreased if all proposed interventions were accepted.

\section{Introduction}

As the population in developed countries is aging, more psychotropics are used in clinical practice, increasing the chance of inappropriate prescribing and polypharmacy (patients taking five or more substances daily). Polypharmacy can be medically unnecessary and increase the risks of potentially inappropriate medications (PIMs) [1, 2, 3]. PIMs are medications for which the risks of use exceed the desired treatment benefits and which can cause harm, treatment failure, or increased treatment costs. Their use is widespread in elderly patients $[4,5,6,7,8]$. There are established PIMs lists used in clinical practice, such as the PRISCUS and the Beers list, or the START/STOPP criteria [9, 10, 11]. Lau et al. demonstrated that patients with PIMs in pharmacotherapy (as defined by the combined version of the Beers list) had a higher likelihood of hospitalization due to treatment with PIMs over one year (odds ratio $(\mathrm{OR})=1.73$; 95\%; confidence interval $(\mathrm{Cl}) 1.14-2.60)$ and higher mortality $(\mathrm{OR}=1.89 ; 95 \% \mathrm{Cl} 1.47-2.44)$, which increased treatment costs. A higher risk for hospitalization $(\mathrm{OR}=1.80,95 \% \mathrm{Cl} 1.34-2.41)$ and higher mortality $(\mathrm{OR}=1.28,95 \% \mathrm{Cl} 1.05-1.55)$ were also observed in nursing home residents exposed to PIMs for at least two months [5].

Among PIMs, an Austrian study of nursing home settings found psychotropics to be the most common [4]. The study included 48 out of 50 nursing homes and 1,844 out of 2,005 residents. It found that 1,014 $(55 \%)$ of the residents had at least one psychotropic PIM. The most commonly prescribed PIM was the antipsychotic prothipendyl ( $25.9 \%$ of the residents) [4]. Lee et al. conducted a similar study on the elderly $(\mathrm{M}=81, \pm 4.3)$ with depressive symptoms. The median number of medications used was 6 (range 1-21 medications). The most commonly prescribed PIMs (Beers list) were amitriptyline, dextropropoxyphene, quinine, and benzodiazepines. Almost half (49\%) of the participants were prescribed at least one PIM [11]. In addition, a U.S. study shows that antipsychotics already on PIM lists may be connected with 
higher mortality than previously known [13]. Its authors conducted a retrospective cohort study using the 1999-2008 national data from the U.S. Department of Veterans Affairs for dementia patients aged 65 or above who began outpatient treatment with either an antipsychotic (risperidone, olanzapine, quetiapine, or haloperidol) or valproic acid and its derivatives (as a non-antipsychotic comparison). The total sample included 33,604 patients, and individual drug groups were compared for 180-day mortality rates. In covariate-adjusted intent-to-treat analyses, haloperidol was associated with the highest mortality rates (relative risk $=1.54,95 \% \mathrm{Cl} 1.38-1.73$ ) followed by risperidone (reference), olanzapine (relative risk $=0.99$, $95 \% \mathrm{Cl} 0.89-1.10$ ), valproic acid and its derivatives (relative risk $=0.91,95 \% \mathrm{Cl} 0.78-1.06$ ), and quetiapine (relative risk $=0.73,95 \% \mathrm{Cl} 0.67-0.80$ ) [13].

These three studies demonstrate that elderly MHP patients are especially vulnerable to PIMs and that their treatment should be studied further. Some clinical studies suggest that interventions in the treatment by a clinical pharmacist (CP) may be one of the strategies to reduce PIMs in patients with MHPs $[1,14]$. In a prospective non-randomized pre-post study examining elderly residents with MHPs in a Slovene nursing home, Stuhec et al. report a reduction in the total number of PIMs as well as a significant increase in quality of life measures $(p<0,05)$ after a medical review was provided by a CP $(n=24)$. However, no causal relationships can be established due to the non-randomized pre-post study design as well as the very small sample size $(n=24)$.

This study aimed to determine the prevalence of PIMs and the effect of CP's interventions on the number of PIMs in elderly persons with MHPs in primary care. We hypothesized to confirm a positive association between the acceptance of the CP's recommendations and a lower number of PIMs in patients with MHPs ( $\mathrm{H} 1)$. We also hypothesized a positive association between the full acceptance of the CP's interventions and a lower number of PIMs compared to partial acceptance $(\mathrm{H} 2)$.

\section{Methods}

\section{Study design, inclusion and exclusion criteria, and data collection}

This study examined the data from one Slovenian (European Union) primary setting (Ljutomer Health Centre in the northeast of Slovenia), which caters to approximately 10,000 inhabitants. We selected all patients aged 65 or more (cohort), who were receiving polypharmacy ( 5 or more medications at the same time), had at least one mental disorder diagnosis according to ICD-10 standards and were prescribed at least one psychotropic drug for it, and were referred to the psychiatric CP by general practitioners (GPs) for a medical review between 1.1.2015 and 1.6.2017 [15].

A psychiatric $\mathrm{CP}$ with eight years of work experience in a psychiatric hospital and an ambulatory setting worked within a primary care setting and, upon the request of a GP, would prepare a medical review (as standardized by the Slovenian Pharmacy Act; for a detailed structure of the reviews check our other papers) $[1,14,16]$. The psychiatric $\mathrm{CP}$ conducted a medical review and provided medication 
recommendations to GPs. They then approved or rejected the recommendations and implemented them at the patient's next visit to the GP (up to two months from the medical review). The clinical pharmacist communicated with GPs through the medical review and by phone if necessary.

This service was initiated by the Institute for Health Insurance of Slovenia due to prevalent excessive polypharmacy in Slovenia and was adopted in the Slovenian healthcare system in 2016 [1]. The data for this study was collected by AN, who checked all medical reviews and patients' charts together with MS. AN also identified PIMs from medical reviews and patient charts using the Beers and the PRISCUS list [9, 10]. Researchers and patients were not blinded because the study has included a retrospective design.

\section{Patient selection}

This study did not use a randomization process and included all patients during the study period who fulfilled the inclusion criteria and for whom there was no missing data. For each study participant, we retrieved both paper and electronic records of their medical list at three-time points. The first was before the CP's recommendations, the second was at the participants' first visit to the GP after the CP's recommendations (up to two months after the recommendations were given to the GP), and the third point was at six months after the first post-review visit to the GP. Aside from information in the medical reviews, no clinical outcome measures were considered in this study. The STROBE Statement checklist was used to include all required items for a cohort study report, and a flow chart was also created [17]. We recorded the number of PIMs for each participant's medication list at the time points for repeatedmeasures testing and the cohort study.

\section{Cohort design}

The cohort in this study consisted of all included patients who fulfilled the inclusion and exclusion criteria. The cohort was divided into two groups using the full or partial GP's acceptance of the recommendations as an exposure factor: group N\#1 included participants for whom all proposed CP's interventions were accepted (exposed), and group N\#2 included participants for whom not all proposed

CP's interventions were accepted (non-exposed). Afterward, the ORs were compared between two groups (exposed/non-exposed) according to the Beers and the PRISCUS list separately $[9,10]$.

\section{Statistical analysis}

Descriptive statistics were calculated to describe the sample. A dependent samples (paired) t-test was used to compare the participant's results between 2-time points (before the interventions and up to two months after the intervention). If the sample values were not normally distributed, the nonparametric Wilcoxon signed-rank test was used. To compare the two groups in the cohort, we used ORs with appropriate confidence intervals. The statistical significance threshold was predefined and set at $p<0.05$. A hypothesis-based approach was used in this study for manuscript writing, and two different hypotheses were included ( $\mathrm{H} 1$ = number of PIMs after the CP's interventions decreased; $\mathrm{H} 2=\mathrm{GP}$ 's full acceptance of the CP's interventions decreased the likelihood of PIMs) [18]. Analyses were carried out with the Statistical Package for Social Science 22.0 for Windows ${ }^{\circledR}$ (SPSS). 
This study was approved by the National Medical Ethics Committee of the Republic of Slovenia in 2017 (number 0120-489/2017/4). All experimental protocols were approved by a National Medical Ethics Committee of the Republic of Slovenia in 2017 (number 0120-489/2017/4). All methods were carried out in accordance with relevant guidelines and regulations. Informed consent was not necessary because it was a retrospective study and this was approved by the National Medical Ethics Committee of the Republic of Slovenia in 2017 (number 0120-489/2017/4).

\section{Results}

\section{General Results}

Ninety-nine patients were included in this cohort retrospective study (Fig. 1, Flowchart). This study included 60 women (60.0\%) and 39 men (40.0\%). The average age of the patients was 79.4 years (SD = 7.92). In total, the participants were treated with 1070 different medications (average 10.8 medications, median 11). Out of those, 285 (26.6\%) medications were psychotropics, and the average number of psychotropics per patient was 2.9 (median 3).

The CP proposed a total of 402 interventions (discontinuations, adjustments, initiations). 4.1 interventions (median 4) per patient, out of which 164 interventions were related to psychotropics (1.7 per patient). In total, the CP proposed 258 discontinuations (an average of 2.6 discontinuations per patient) of existing medicines, representing $64.2 \%$ of all proposed interventions, 109 initiations (an average of 1.1 initiations), and 35 dose adjustments ( 0.35 on average). The highest number of proposed interventions was 11 (in 1 patient), and the CP suggested 4 to 7 interventions in more than half of the patients.

After the CP's interventions, the total number of medications decreased by $9.2 \%$ from 1070 to $972(p=$ $0.000, p<0.05$ ), decreasing the average number of medications per patient from 10.8 to 9.8 . The number of medications decreased for 50 patients, remained the same for 47 , and increased in 2 patients. In total, the GPs accepted 177 interventions (44.0\% of all proposed interventions), out of which 73 interventions were related to psychotropics. In most cases, the post-intervention pharmacotherapy did not change in 6 months after the intervention, except in two cases in which the pharmacotherapy was switched back to the pre-intervention treatment (from $150 \mathrm{mg}$ venlafaxine back to $75 \mathrm{mg}$ daily in one case and methyldigoxine reinitiation in a participant treated with bisoprolol).

\section{Potentially inappropriate medications in the elderly}

Before the medical review, 109 PIMs, as defined by the PRISCUS list, were found. These PIMs represented $10.2 \%$ of the prescribed medications (1.1 PIM per patient) (Fig. 2). $69.7 \%$ patients $(n=69)$ received at least one PIM. The most frequent PIMs were sedatives and hypnotics (72 PIMs, $66.1 \%$ of the PIMs), followed by antipsychotics (17 PIMs, 15.6\%) and antiarrhythmics (8 PIMs, 7.3\%).

Using the Beers list, we identified 220 PIMs (Fig. 3), representing $20.6 \%$ of all medications (2.2 PIMs per patient). At least one PIM (Beers list) was found in $92.9 \%$ of the patients $(n=92)$. Eighty-eight PIMs were 
connected with benzodiazepines and z-hypnotics (40.0\% of all PIMs using the Beers list), followed by gastrointestinal medications (54 PIMs, 24.5\%) and antipsychotics with 37 PIMs (16.8\%).

After the interventions and GPs acceptance/rejections, the number of PIMs (PRISCUS list) decreased by $21.1 \%$ from 109 to $86(p=0,000, p<0.05)$, reducing the average number of PIMs per patient from 1.1 to 0.89. The number of PIMs defined through the Beers list decreased by $17.3 \%$ from 220 to 182 ( $p=0,000$, $p<0.05$ ), resulting in a decrease in the average number of PIMs per patient, 2.2 to 1.8. The results show a statistically significant reduction $(p<0.05)$ in PIMs after the interventions, using either the PRISCUS or Beers list.

\section{Cohort study results (exposure/non-exposure)}

The cohort study included 99 patients in the exposure/non-exposure analysis (Fig. 4). There was no statistically significant difference between both groups in terms of age $(p=0.107, p>0.05)$ and the total number of medications per patient before the intervention $(p=0.586, p>0.05)$, but there was a statistically significant difference between both groups by gender $(p=0.017,0<0.05)$. In $34.8 \%$ or $52.2 \%$ of the patients (PRISCUS and Beers list, respectively) the total number of PIMs decreased in group N\#1 (full acceptance of the CP's recommendations) compared with $15.8 \%$ or $22.4 \%$ of the patients in group N\#2 (PRISCUS and Beers list, respectively). The OR for PRISCUS list PIMs was 2.8 (Cl 1.0-8.2) and $3.8(\mathrm{Cl}$ 1.4-10.1) for Beers list PIMs. The GP's full acceptance of the CP's interventions, therefore, decreased the likelihood of PIMs, partially confirming our second hypothesis (the $\mathrm{Cl}$ for PRISCUS list includes 1), because the results for the PRISCUS list show a positive trend but are not statistically significant.

\section{Discussion}

This study is the first retrospective long-term cohort study assessing the impact of the CP's interventions on the number of PIMs through a medical review service in elderly patients with MHPs in Central Europe, which opens a new window for collaborative care implementation within primary care in Slovenia and broader in Europe. This study provides three key findings.

Firstly, the CP's interventions led to fewer medications per patient, as in previous studies $[1,14,16,19]$. The service examined in this study may reduce the number of medications per patient and, by extension, the frequency of polypharmacy and its related risks. $[1,14,16]$. This reduction was found although the GPs accepted less than $50 \%$ of proposed interventions, a rate comparable to a previous study in primary care [1]. One reason for a relatively low acceptance rate could be the lack of a follow-up by a CP after the initial medical review, which was not done in the study period. Patient monitoring by $\mathrm{CP}$ is already a practice in the U.S. model of collaborative care, and CP in some countries can also prescribe medications. Because the health system in Slovenia (and elsewhere) faces a lack of GPs and psychiatrists, expanding the role of CP in the treatment of MHPs may be beneficial. The relatively low acceptance rate could also be because patients with MHPs are also managed by psychiatrists, who in some cases rejected the proposed pharmacotherapy changes. This contrasts with a 2015 study by Stuhec et al. [19], where a similar service had very high acceptance rates $(88.0 \%)$ in a psychiatric hospital, in which the CP was also 
working daily in hospital wards (e. g. doing daily roundings). Higher acceptance rates could likely be achieved in the setting we studied through increased collaboration between CPs with psychiatrists, which was not examined in this study. These results are also interesting in terms of different intervention types because the $\mathrm{CP}$ suggested many treatment initiations, in one case suggesting clozapine initiation in line with the treatment guidelines [21, 22]. In addition, almost all pharmacotherapy received by the patients remained the same six months after the CP's interventions, implying that they were positive (positive adherence) and did not cause adverse events. However, in two cases, the pharmacotherapy was switched back in 6 months, warranting further studies on the potential complications of returning to previous therapy.

The second significant result is that the number of PIMs after the CP's interventions decreased as hypothesized for both the PRISCUS and the Beers list. Therefore we can confirm our first hypothesis $(\mathrm{H} 1)$. The total number of PIMs (PRISCUS list) decreased by $21.1 \%$. The most considerable PIMs reduction occurred with hypnotics and sedatives (e.g., zolpidem, bromazepam, alprazolam, diazepam) (Fig. 2), which is again in line with the recommendations because benzodiazepines should be avoided in elderly patients due to their negative effect on falls and cognitive decline $[4,9,10,11]$. In cases of depression, anxiety disorders, and long-term treatment of insomnia, antidepressants (e.g., mirtazapine, trazodone, SSRIs) are suggested in particular, which was mostly proposed in this study, meaning that these interventions also lead to better treatment guidelines adherence $[4,9,10,11]$. Other important PIMs observed in this study were connected with antipsychotics, clozapine, olanzapine, and haloperidol.

Interestingly, the CP suggested clozapine initiation in line with the guidelines, which were accepted by the psychiatrist and GP $[21,22]$. Another important finding is the reduction of the number of patients treated with haloperidol, which is connected with very high mortality and extrapyramidal adverse effects and for which other antipsychotics (e.g., quetiapine) are suggested as a substitute $[9,10,13]$. In line with the Beers list, the use of proton pump inhibitors, zolpidem, quetiapine, bromazepam, and diazepam was reduced. Similar results also apply to the PRISCUS list. Proton pump inhibitors are often used without long-term indication, which can lead to several adverse events, including falls [10]. Quetiapine is often used for insomnia against the treatment guidelines, and the CP suggested different medications (e.g., mirtazapine and trazodone) [23]. These results are positive, but many PIMs remained in the patients' pharmacotherapy, so additional monitoring and interventions could further reduce the risks, which may increase the acceptance rates by GPs. These results are similar to an Austrian study that found antipsychotics and benzodiazepines to be most frequently connected with PIMs; primary care could be improved through collaborative care that includes a clinical pharmacists' review service [4].

The third set of results are those of the cohort analysis in our study. The results were only statistically significant using the Beers list, which could be because of the low sample size, and therefore we can only partially confirm our second hypothesis $(\mathrm{H} 2)$. These results also demonstrate how the two used PIM criteria differ. In full recommendation acceptance cases, the number of PIMs decreased, opening a new question about further service implementation. These results are in line with previous studies on different populations that found improved treatment adherence because of accepted interventions $[1,16]$. 
Our study also has many limitations that should be discussed. The sample size we used was relatively small and not calculated (high risk of type II error), which can have an impact on the statistical significance of the results as well as the calculation of Cls of the ORs, so the study should be replicated with a larger sample and calculated sample size which would reduce risk of bias. Our study also had a selection bias (e.g., no randomization) because the patients were selected by the GPs (who referred them to the CP's service). Furthermore, we included all patients with MHPs according to the ICD-10, which means that the population was very heterogeneous. On the other hand, the minimal exclusion criteria may mean that our results have higher ecological validity, which is informative for service implementation and clinical implication. Another significant limitation is the lack of direct collaboration between the CP, psychiatrist, and GP, which might account for a relatively low number of accepted interventions. Due to the retrospective study design, we also did not have contact with the study participants, and clinical outcomes were not measured. Despite its limitation, this study contributes new insights on collaborative care that includes a psychiatric CP and expands the knowledge on this type of service in Central Europe in this vulnerable population.

Collaborative care that included a CP reduced the number of PIMs as defined by both the PRISCUS and the Beers list, where almost all interventions were maintained. The ORs for PIMs were also smaller in cases of full intervention acceptance. These results confirm the positive effects of CP's interventions within this field in clinical practice. The results are also the first published in Central Europe, although they should be replicated by studies with larger sample sizes and fewer limitations. These results could also inform future research on CPfocused collaborative care in primary care settings in elderly patients with MHPs in Central Europe.

\section{Declarations}

\section{Acknowledgements}

None

\section{Author Contributions Statement}

Conceived and designed the study: MS, AN. Collected the data: AN. Analyzed the data: AN, MS. Drafted the manuscript: MS, AN. Commented the draft of the manuscript: all authors. Read and approved the final version of the manuscript: all authors.

\section{Funding}

The authors declare that they have no conflicts of interest with regard to this work. The author MS acknowledge the financial support from the Slovenian Research Agency for manuscript writing (research core funding No. P3-0036, Biopsychosocial model of quality of life).

\section{Competing interests}


The authors declare no competing interests.

\section{References}

1. Stuhec, M., Gorenc, K. \& Zelko, E. Evaluation of a collaborative care approach between general practitioners and clinical pharmacists in primary care community settings in elderly patients on polypharmacy in Slovenia: a cohort retrospective study reveals positive evidence for implementation. BMC Health Serv Res. 19 (1), 118 (2019).

2. Stuhec, M. \& Gorenc, K. Clinical pharmacist interventions in elderly patients in primary care treated with polypharmacy and psychotropics: Observational retrospective study. European Neuropsychopharmacology. 29, S545-S546 (2019).

3. Rieckert, A. et al. Polypharmacy in older patients with chronic diseases: a cross-sectional analysis of factors associated with excessive polypharmacy. BMC Fam Pract. 19, 113 (2018).

4. Mann, E. et al. Prevalence and associations of potentially inappropriate prescriptions in Austrian nursing home residents: secondary analysis of a cross-sectional study. Wien Klin Wochenschr. 125, 180-188 (2013).

5. Lau, D. T., Kasper, J. D., Potter, D. E., Lyles, A. \& Bennett, R. G. Hospitalization and death associated with potentially inappropriate medication prescriptions among elderly nursing home residents. Arch Intern Med. 165, 68-74 (2005).

6. Fick, D. M., Mion, L. C., Beers, M. H. \& Waller, J. Health outcomes associated with potentially inappropriate medication use in older adults. Res Nurs Health. 31, 42-51 (2008).

7. Albert, S. M., Colombi, A. \& Hanlon, J. Potentially inappropriate medications and risk of hospitalization in retirees: analysis of a US retiree health claims database. Drugs Aging. 27, 407-415 (2010).

8. Ruggiero, C. et al. Potentially inappropriate drug prescriptions and risk of hospitalization among older, Italian, nursing home residents: the ULISSE project. Drugs Aging. 27, 747-758 (2010).

9. Amann, U., Schmedt, N. \& Garbe, E. Prescribing of potentially inappropriate medications for the elderly: an analysis based on the PRISCUS list. Dtsch Arztebl Int. 109, 69-75 (2012).

10. Fick, D. M. et al. Updating the Beers criteria for potentially inappropriate medication use in older adults: results of a US consensus panel of experts. Arch Intern Med. 163, 2716-2724 (2003).

11. O'Mahony, D., O'Sullivan, D., Byrne, S., O'Connor, M. N. \& Ryan, C. \& Gallagher, P. STOPP/START criteria for potentially inappropriate prescribing in older people: version 2. Age Ageing. 44, 213-218 (2015).

12. Lee, D. et al. Potentially inappropriate medication use: the Beers' Criteria used among older adults with depressive symptoms. J Prim Health Care. 5, 182-190 (2013).

13. Kales, H. C. et al. Risk of mortality among individual antipsychotics in patients with dementia. Am J Psychiatry. 169, 71-79 (2012). 
14. Stuhec, M., Bratović, N. \& Mrhar, A. Impact of clinical pharmacist's interventions on pharmacotherapy management in elderly patients on polypharmacy with mental health problems including quality of life: A prospective non-randomized study. Sci Rep. 9, 16856 (2019).

15. 10th revision of the International Statistical Classification of Diseases and Related Health Problems (ICD), a medical classification list by the World Health Organization (WHO) (accessed on 9.11.2018), https://www.who.int/classifications/icd/en/.

16. Stuhec, M. \& Gorenc, K. Positive impact of clinical pharmacist interventions on antipsychotic use in patients on excessive polypharmacy evidenced in a retrospective cohort study. Global Psychiatry. 2, 155-163 (2019).

17. von Elm, E. et al. STROBE Initiative. The Strengthening the Reporting of Observational Studies in Epidemiology (STROBE) statement: guidelines for reporting observational studies. J Clin Epidemiol. 61, 344-349 (2008).

18. Heun, R. How to write a scientific paper: A hypothesis-based approach. Global Psychiatry. 1, 3-6 (2018).

19. Hajjar, E. R., Cafiero, A. C. \& Hanlon, J. T. Polypharmacy in elderly patients. Am J Geriatr Pharmacother. 5, 345-351 (2007).

20. Stuhec, M. Pharmacotherapy review as a safety and cost tool in patients management in Slovenian psychiatric hospital. V: abstracts of the 27th ECNP congress, Berlin, Germany, 18-21 October 2014. Eur Neuropsychopharmacol. 24, S735-6 (2014).

21. Stahl, S. M. Emerging guidelines for the use of antipsychotic polypharmacy. Rev Psiquiatr Salud Ment. 26, 97-100 (2013).

22. Howes, O. D. et al. Adherence to treatment guidelines in clinical practice: study of antipsychotic treatment prior to clozapine initiation. Br J Psychiatry. 201, 481-485 (2012).

23. Wilson, S. J. British Association for Psychopharmacology consensus statement on evidence-based treatment of insomnia, parasomnias and circadian rhythm disorders. J Psychopharmacol. 24, 15771601 (2010).

\section{Figures}




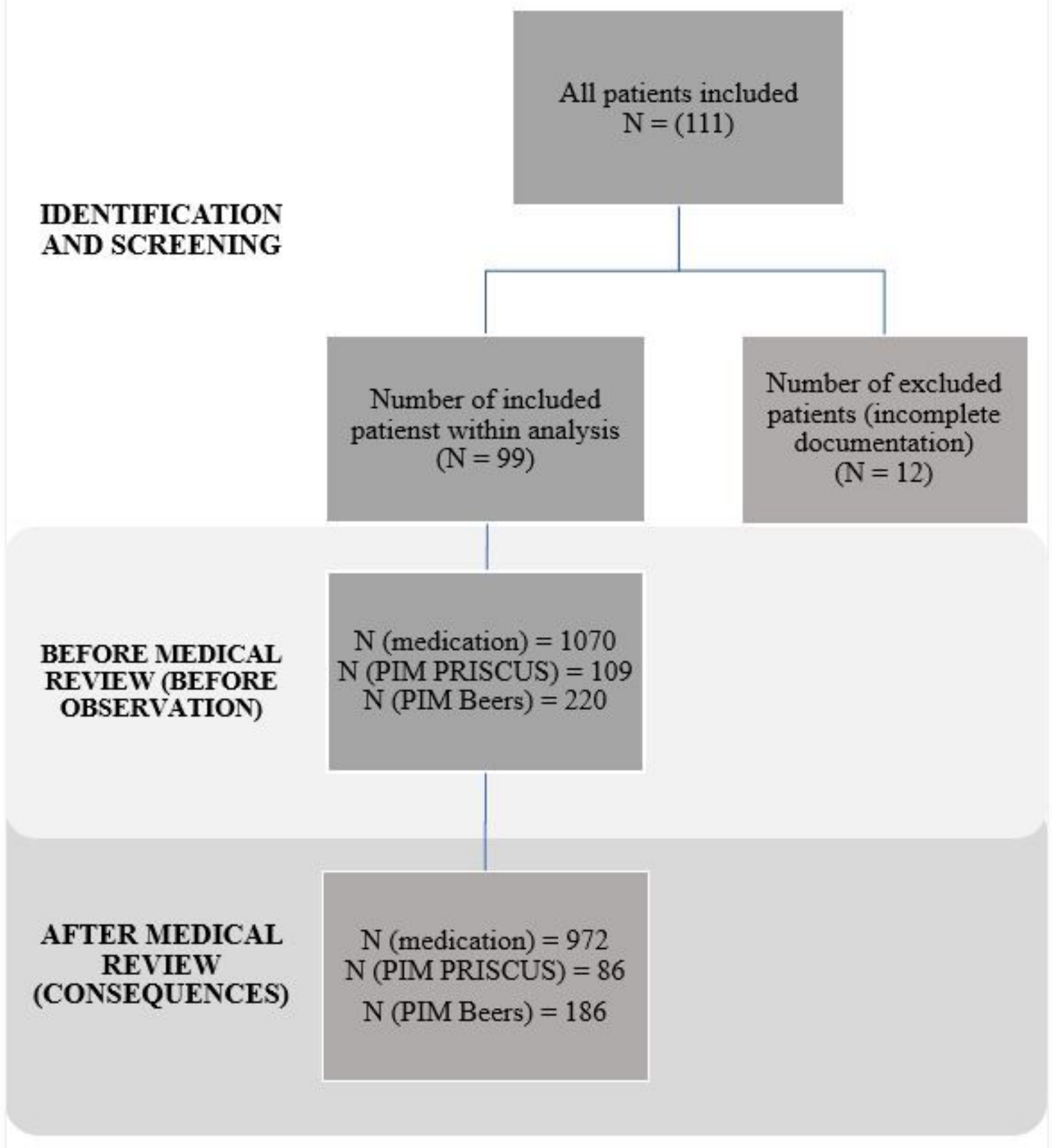

Figure 1

Flowchart. 


\section{Priscus list: Comparison of the PIM number before and after the medical review}

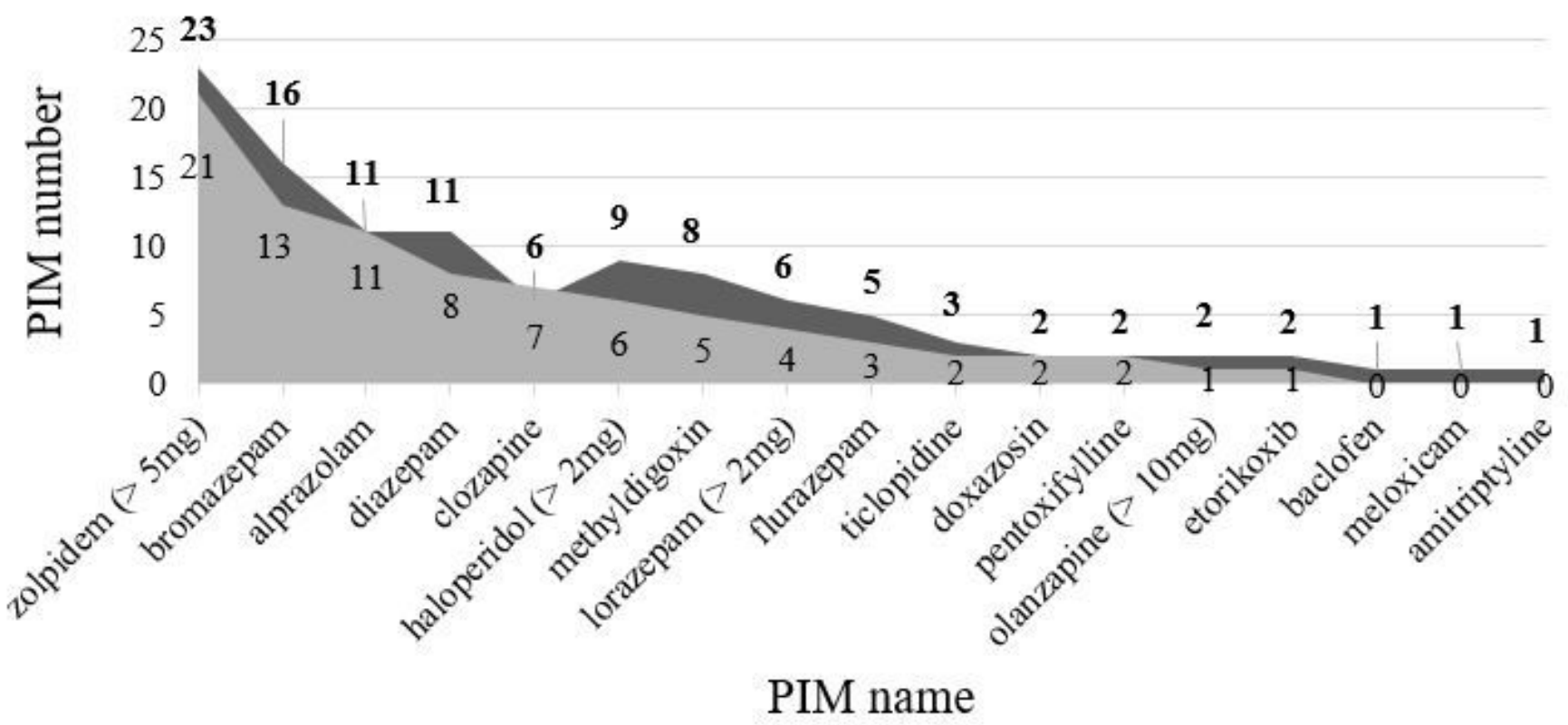

Before medical review $\quad=2$ months after medical review

Figure 2

The total number of PIMs before and after the medical review (PRISCUS list). 


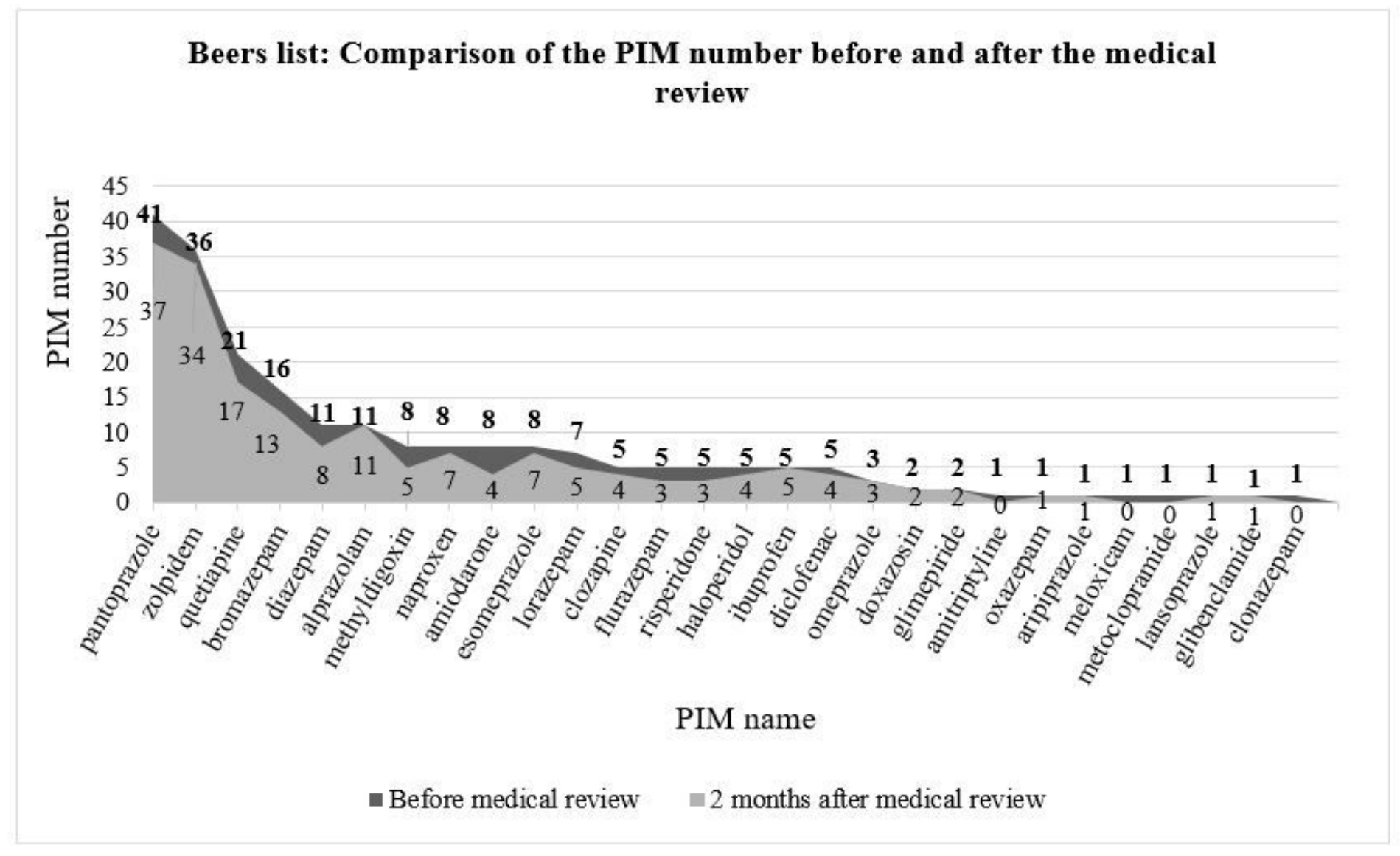

Figure 3

The total number of PIMs before and after the medical review (Beers list). 


\section{IDENTIFICATION AND SCREENING}

\section{EXPOSURE FACTOR}

\section{CONSEQUENCES (OUTCOMES)}

$$
\begin{gathered}
-99 \text { patients } \\
-60.0 \% \text { women, } 39.4 \% \text { men } \\
\text { - average age } 79.4 \text { y } \\
\text { - avegare number of medications } 10.8 \text { before } \\
\text { review }
\end{gathered}
$$

Exposured (all interventions were accepted)

$$
-23 \text { patients }
$$

$-39.1 \%$ women and $60.9 \%$ men

- average age $77,8 \mathrm{y}$

- N of medications 10.0 after medical review

- N of PIMs 0.91 (Priscus list) after medical review

- N of PIMs 1.7 (Beers) after medical review
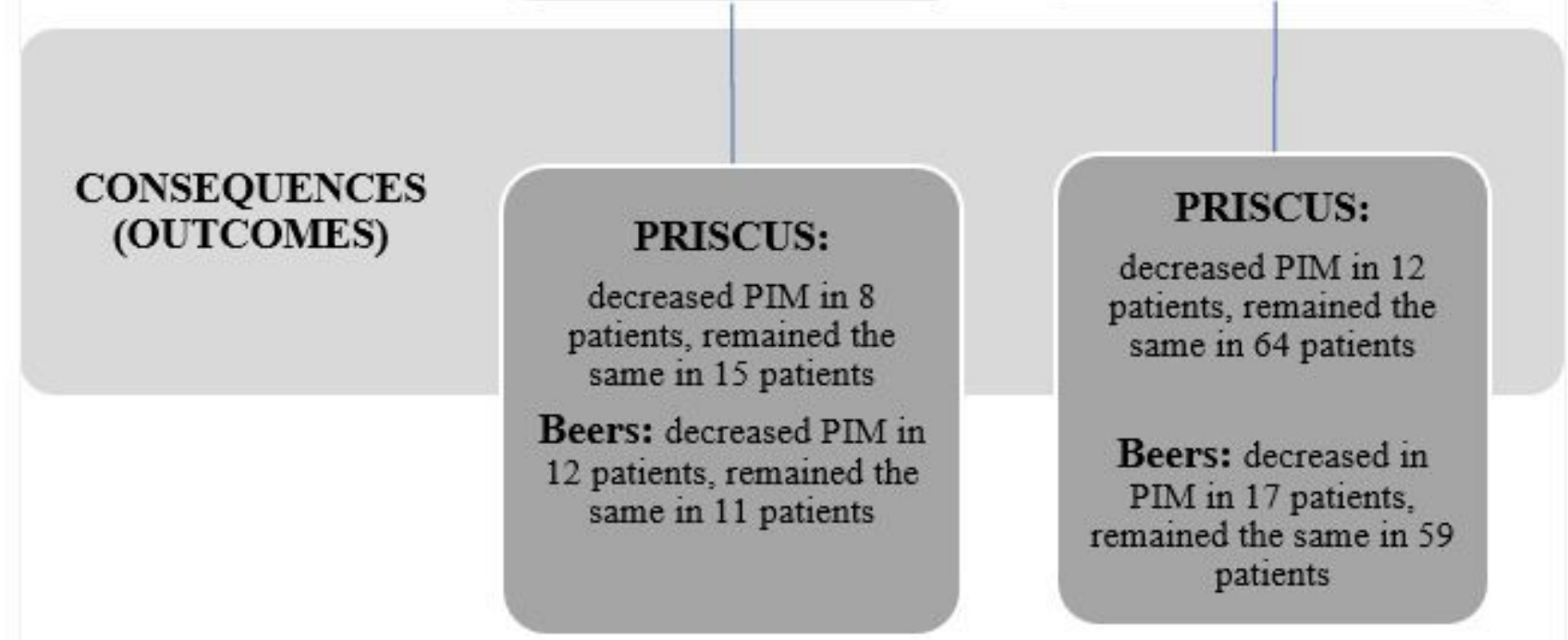

\section{Figure 4}

Summary of the results of cohort study including both subgroups (flowchart). 